\title{
ARTICLE \\ Reduced fractional anisotropy in depressed patients due to childhood maltreatment rather than diagnosis
}

Susanne Meinert ${ }^{1}$, Jonathan Repple ${ }^{1}$, Igor Nenadic ${ }^{2}$, Axel Krug ${ }^{2}$, Andreas Jansen ${ }^{2,3}$, Dominik Grotegerd ${ }^{1}$, Katharina Förster ${ }^{1}$, Verena Enneking ${ }^{1}$, Katharina Dohm ${ }^{1}$, Simon Schmitt ${ }^{2}$, Frederike Stein ${ }^{2}$, Katharina Brosch ${ }^{2}$, Tina Meller ${ }^{2}$, Ronny Redlich ${ }^{1}$, Joscha Böhnlein ${ }^{1}$, Lisa Sindermann ${ }^{1}$, Janik Goltermann (D) ${ }^{1}$, Elisabeth J. Leehr ${ }^{1}$, Nils Opel ${ }^{1,4}$, Leni Aldermann ${ }^{1}$, Andreas Reuter ${ }^{1}$, Ricarda I. Schubotz ${ }^{5}$, Tim Hahn ${ }^{1}$, Tilo Kircher $^{2}$ and Udo Dannlowski ${ }^{1}$

Reduced fractional anisotropy (FA) associated with Major Depressive Disorder (MDD) overlaps anatomically with effects of childhood maltreatment experiences. The aim of this study was, therefore, to replicate the negative effect of childhood maltreatment on white matter fiber structure and to demonstrate, that alterations in MDD might be partially attributed to the higher occurrence of childhood maltreatment in MDD. Two independent cohorts (total $N=1256$ ) were investigated in a diffusion tensor imaging study: The Münster Neuroimaging Cohort (MNC, $N=186 \mathrm{MDD}, N=210$ healthy controls, HC) as discovery sample and the Marburg-Münster Affective Disorders Cohort Study (MACS, N=397 MDD, N=462 HC) as replication sample. The effects of diagnosis (HC vs. MDD) and Childhood Trauma Questionnaire (CTQ) scores on FA were analyzed. A main effect of diagnosis with higher FA in MDD patients compared with HC was found in the MNC ( $\left.p_{\mathrm{FWE}}=0.021\right)$, but not in the MACS $\left(p_{\mathrm{FWE}}=0.52\right)$ before correcting for CTQ. A significant negative correlation of FA with CTQ emerged in both cohorts (MNC: $p_{\mathrm{FWE}}=0.006, \mathrm{MACS}: p_{\mathrm{FWE}}=$ 0.012 ) in several tracts previously described in the literature. No CTQ $\times$ diagnosis interaction could be detected. Any main effect of diagnosis was abolished after correcting for CTQ (MNC: $p_{\mathrm{FWE}}=0.562, \mathrm{MACS}$ : $p_{\mathrm{FWE}}=0.115$ ). No differences in FA between MDD and $\mathrm{HC}$ could be found after correcting for childhood maltreatment, suggesting that previously reported group differences might be attributed partially to higher levels of maltreatment experiences in MDD rather than diagnosis itself. Furthermore, a well-established finding of reduced FA following childhood maltreatment experiences was replicated.

Neuropsychopharmacology (2019) 44:2065-2072; https://doi.org/10.1038/s41386-019-0472-y

\section{INTRODUCTION}

Childhood maltreatment, including physical, and emotional neglect as well as physical, emotional, and sexual abuse, is highly prevalent [1]. It is a risk factor for the development of major depressive disorder (MDD) [2-5], accounting for 54\% of the population attributable risk for depression [6]. It is associated with lower responding rate to psychopharmacological treatment and higher likelihood of chronicity in MDD [7-11]. Furthermore, childhood maltreatment is associated with immunological, endocrine, and epigenetic processes, as well as brain structure and function [4, 12-14]. These changes could be initially adaptive reactions to a dysfunctional environment, increasing the vulnerability to depressive psychopathology $[15,16]$.

MDD and childhood maltreatment have been associated with adverse changes in various overlapping white matter regions: in the inferior fronto-occipital fasciculus [17-20], the uncinate fasciculus $[16,18,19]$, the thalamic radiation $[17,18,20,21]$, the corona radiata or corticospinal tract [19, 21, 22], the longitudinal fasciculi $[15-17,19,20]$ the cingulum bundle $[19,20]$ and the corpus callosum $[7,16,17,19,21,23,24]$. This anatomic overlap strengthens the idea of a close link between stressful life events in early childhood and MDD. However, it also advises caution, as neurobiological alterations typically ascribed to MDD disease status could be explained to some extent to the higher occurrence of childhood maltreatment experiences in MDD patients [24]. To understand the brain structural correlates of MDD these effects have to be distinguished.

Previous studies already suggested that differences in gray matter volume and functional amygdala responsiveness between patients with MDD and healthy controls $(\mathrm{HC})$ might rather be a function of childhood maltreatment than of MDD itself $[25,26]$, advising to correct for its influence in future studies investigating MDD. As it becomes increasingly evident that childhood maltreatment experiences influence a complex neural network, investigating interconnecting fibers is a matter of particular interest.

Diffusion tensor imaging (DTI) uses estimations of fractional anisotropy (FA) to quantify fiber structure in a noninvasive population-based approach. FA measures directional diffusion, taking values from zero (=isotropic) to one (=anisotropic) constrained along one axis). As FA is influenced by myelination, axon density, axon diameter, and the permeability of the brain, it is often interpreted to reflect axonal damage [27, 28]. However, the number of fibers, fiber crossings and general fiber orientation can also influence FA in healthy fiber structure [29].

\footnotetext{
${ }^{1}$ Department of Psychiatry, University of Münster, Münster, Germany; ${ }^{2}$ Department of Psychiatry and Psychotherapy, University of Marburg, Marburg, Germany; ${ }^{3}$ Core-Unit Brain Imaging, Faculty of Medicine, University of Marburg, Marburg, Germany; ${ }^{4}$ Interdisciplinary Centre for Clinical Research (IZKF), University of Münster, Münster, Germany and ${ }^{5}$ Department of Psychology, University of Münster, Münster, Germany Correspondence: Udo Dannlowski (dannlow@uni-muenster.de)
}

Received: 4 June 2019 Revised: 13 July 2019 Accepted: 23 July 2019 Published online: 5 August 2019 
Table 1. Sociodemographic and clinical characteristics of the cohorts

\begin{tabular}{|c|c|c|c|c|c|c|}
\hline Characteristic & \multicolumn{3}{|l|}{ MNC } & \multicolumn{3}{|l|}{ MACS } \\
\hline \multicolumn{7}{|l|}{ Sociodemographic } \\
\hline Age, years & $38.66 \pm 11.68$ & $38.61 \pm 10.40$ & $0.966^{c}$ & $37.31 \pm 13.47$ & $36.74 \pm 12.72$ & $0.522^{c}$ \\
\hline \multicolumn{7}{|l|}{ Questionnaires } \\
\hline CTQ sum & $47.11 \pm 17.36$ & $36.64 \pm 9.08$ & $<0.001^{c}$ & $47.57 \pm 16.49$ & $34.17 \pm 9.21$ & $<0.001^{c}$ \\
\hline CTQ emotional neglect & $13.55 \pm 5.42$ & $10.62 \pm 3.89$ & $<0.001^{c}$ & $13.83 \pm 5.44$ & $9.15 \pm 3.77$ & $<0.001^{c}$ \\
\hline СТQ physical neglect & $8.17 \pm 3.11$ & $6.91 \pm 2.05$ & $<0.001^{\mathrm{c}}$ & $8.26 \pm 3.30$ & $6.49 \pm 1.96$ & $<0.001^{\mathrm{c}}$ \\
\hline CTQ sexual abuse & $6.44 \pm 3.81$ & $5.32 \pm 1.17$ & $<0.001^{c}$ & $6.54 \pm 3.54$ & $5.34 \pm 1.50$ & $<0.001^{c}$ \\
\hline CTQ emotional abuse & $11.40 \pm 6.44$ & $7.83 \pm 3.37$ & $<0.001^{c}$ & $11.67 \pm 5.27$ & $7.45 \pm 3.22$ & $<0.001^{c}$ \\
\hline
\end{tabular}

Reductions of FA in MDD compared with $\mathrm{HC}$ in the inferior fronto-occipital fasciculi, the uncinate fasciculi, the thalamic radiation, the corticospinal tract, the longitudinal fasciculi, the cingulum bundle, and the corpus callosum are expected (main effect diagnosis; analysis 1.1, analysis 2.1). Further, a reduction of FA associated with increased burden of maltreatment should be present in the same fibers (main effect maltreatment; analysis 1.2, analysis 2.2). Differences in FA comparing patients with MDD and $\mathrm{HC}$ should be diminished when controlling for childhood maltreatment (main effect diagnosis; analysis 1.2, analysis 2.2). For internal replication, two independent cohorts were investigated. As the discovery and replication sample differed in clinical characteristics (see below) additional exploratory analyses were conducted (analysis 1.3, analysis 2.3).

\section{MATERIALS AND METHODS}

\section{Participants}

Two independent cohorts-the Münster Neuroimaging Cohort (MNC) [30] and the Marburg-Münster Affective Disorders Cohort Study (MACS) [31] were combined.

First, $N=396$ participants from the MNC, N=186 diagnosed with MDD and $N=210 \mathrm{HC}$ were analyzed. Restrictive inclusion criteria were used in the MNC: All MDD patients were inpatients diagnosed with a severe depressive episode. $\mathrm{HC}$ were recruited through newspaper advertisements.

Second, $N=859$ participants from the MACS $(N=397$ MDD patients suffering from current or partially remitted depressive episode, $N=462 \mathrm{HC}$ ) were selected for analysis (Supplement 1). Participants were recruited through psychiatric hospitals or newspaper advertisements. Inclusion criteria were less strict including mild, moderate or partially remitted MDD episodes on the top of severe depression. Furthermore, patients could be undergoing inpatient, outpatient or no current treatment. The MACS was conducted at two scanning sites-University of Münster and University of Marburg (the magnetic resonance imaging (MRI) quality assurance protocol [32] and the general study description [31] are provided in previous articles).

The MNC was approved by the ethics committee of the Medical Faculty of University of Münster. The MACS was approved by the
Ethics Committees of the Medical Faculties, University of Marburg and University of Münster. All experiments were performed in accordance with the ethical guidelines and regulations. All participants gave written informed consent prior to examination. They received financial compensation for participation after the testing session.

The groups (HC vs. MDD) were matched for age and sex within each sample separately (Table 1). To confirm the psychiatric diagnosis or the lack thereof, the Structural Clinical Interview for DSM-IV-TR (SCID-IV) [33] was assessed by trained personnel. Participants varying in age from 18 to 65 years were recruited. Exclusion criteria in both studies comprised any neurological abnormalities, history of seizures, head trauma or unconsciousness, severe physical impairment (e.g., cancer, epilepsy), hypothyroidism without adequate medication, claustrophobia, color blindness, and general MRI contradictions (e.g., ferromagnetic implants, pregnancy). Lifetime diagnoses of schizophrenia, schizoaffective disorder, bipolar disorder, or substance dependence were excluded.

The German version of the Childhood Trauma Questionnaire (CTQ) [34] was administered to assess adverse early life events. The CTQ is a 28-item retrospective self-report questionnaire covering five types of negative childhood experiences: emotional neglect, physical neglect, emotional abuse, physical abuse, and sexual abuse [35]. The questionnaire uses a fivelevel Likert scale, with higher values reflecting maltreatment of greater magnitude. HC and MDD differed in their overall CTQ score and all subscales (all $p<0.001$, Table 1 ) in the MNC and MACS, respectively. In the following analyses the overall CTQ score was used.

The Hamilton Depression Rating Scale (HDRS) [36] was used to assess depressive symptomatology. The Medication Load Index (MedIndex, Table 2) [37], a composite measure of total medication load reflecting daily dose and number of prescriptions irrespective of active components, was used to measure psychopharmacological medication intake (Supplement 2). MDD patients in the MNC received more psychiatric medication compared with patients in the MACS. However, the two patient cohorts were similar in the number of hospitalizations, number of episodes and comorbid diagnoses (Table 2). 
Table 2. Clinical characteristics, comorbidities, and medication in depressed patients in the Münster Neuroimaging cohort (MNC) and the MarburgMünster Affective Disorders Cohort (MACS)

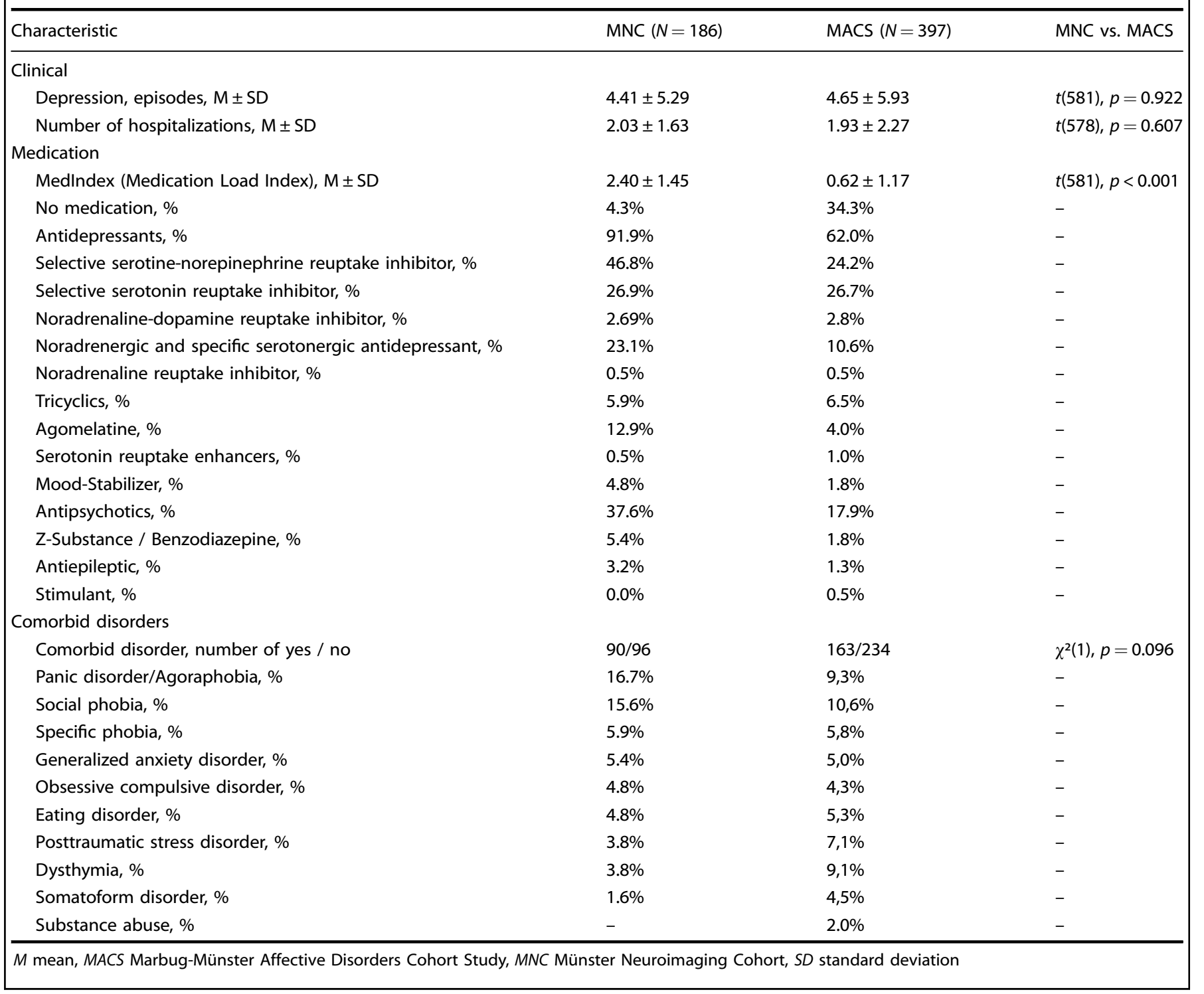

DTI data acquisition

MNC. Data acquisition and preprocessing were performed as reported earlier [30,38]. Data were acquired using a 3T whole body MRI scanner (Gyroscan Intera, Philips Medical Systems, Best, the Netherlands). Thirty-six axial slices, 3.6- $\mathrm{mm}$ thick with no gap were measured with an anisotropic voxel size of $1.8 \times 1.8 \times$ $3.6 \mathrm{~mm}^{3}(\mathrm{TE}=95 \mathrm{~ms}, \mathrm{TR}=9473 \mathrm{~ms})$ in a single-shot echo planar imaging sequence. One nondiffusion-weighted (DW) image $\left(b_{0}=\right.$ 0,3 averages) and $20 \mathrm{DW}$ images with a $b$-value of $1000 \mathrm{~s} / \mathrm{mm}^{2}$ with isotropic gradient directions were acquired.

To ensure data quality, all raw DTI images were visually inspected leading to the exclusion of seven participants prior to matching. If the participants' estimated mean displacement provided by the eddy correct log-file (see below) was greater than three times the standard deviation of all participant's mean displacement, participants were excluded. Thus, five participants were excluded prior to matching. DTIPrep [39] was not used as the MRI scanner and software versions were not compatible.

MACS. Data were acquired using a $3 \mathrm{~T}$ whole body MRI scanner (Marburg: Tim Trio, Siemens, Erlangen, Germany; Münster: Prisma,
Siemens, Erlangen, Germany) using a GRAPPA acceleration factor of 2. Fifty-six axial slices, 2.5-mm thick with no gap, were measured with an isotropic voxel size of $2.5 \times 2.5 \times 2.5 \mathrm{~mm}^{3}(\mathrm{TE}=90 \mathrm{~ms}$, $\mathrm{TR}=7300 \mathrm{~ms})$. Five non-DW images $\left(b_{0}=0\right)$ and $2 \times 30 \mathrm{DW}$ images with a $b$-value of $1000 \mathrm{~s} / \mathrm{mm}^{2}$ were acquired.

For quality control the open-source software DTIPrep [39] was used with default options. DTIPrep detects artifacts caused by eddy currents, head motion, bed vibration and pulsation, venetian blind artifacts, as well as slice-wise and gradient-wise intensity inconsistencies. In case of artifacts, individual images from one participant were omitted from further analyses, with $>20 \%$ of omitted images per participant resulting in the exclusion of that participant (Supplement 1). The included participants had 64.33 images on average ( $S D=1.12$, range: $55-65$ ).

\section{Image processing}

Preprocessing and analysis were performed with FSL5.0.10 (http://fsl. fmrib.ox.ac.uk/fsl/fslwiki/, FMRIB, Oxford Center for Functional MRI of the Brain, University of Oxford, Department of Clinical Neurology, John Radcliffe Hospital, Oxford, United Kingdom) [40-42]. The DW images were corrected for eddy and motion artifacts; $b$-vectors were 
rotated after eddy correction. The first $b_{0}$ was used as reference for alignment following automated skull stripping using FMRIB's brain extraction tool [43] before diffusion tensor estimation using "dtifit" within FMRIB's Diffusion Toolbox [44].

\section{Analysis}

Demographic data were analyzed using IBM SPSS Statistics 25 (SPSS Inc., Chicago, IL, USA). To reduce partial volume effects and registration misalignments, tract based spatial statistics (TBSS) [45] were used. The FMRIB58_FA template was used for registration. Hence, all images were resliced to $1 \times 1 \times 1 \mathrm{~mm}^{3}$ image space. A threshold of 0.2 for the average of all aligned FA images was used to create a white matter skeleton. This skeleton was laid over each participant's registered FA image. The maximum weighted for distance FA orthogonal to the skeleton was moved to skeleton space for group-level comparisons. To test for statistical significance, the nonparametric permutation testing implemented in FSL's "randomize" [46] was used with 5000 permutations. To correct for multiple comparisons, the Threshold-Free Cluster Enhancement (TFCE) with default values optimized for TBSS was used. Significance was determined by using the 95th percentile of the null distribution of permutated input data of the maximum TFCE scores, allowing to correct estimated cluster sizes for the family-wise error (FWE; $p<0.05)$ [47]. MNI coordinates of the peak voxel and cluster sizes were retrieved with FSL's "cluster" tool. Tract labels of significant clusters were extracted using FSL's "atlasquery" and the "JHU White-Matter Tractography Atlas" [48-50]. Mean FA of the significant cluster were extracted using FSL's "fslstats". The total intracranial volume (TIV) was extracted from T1 images using the Computational Anatomy Toolbox (CAT12, http://www.neuro.uni-jena.de/cat, v933). The T1 sequence for MNC [51] and MACS [32] are described elsewhere.

The results focus on FA, as it is most commonly used. However, different DTI metrics as mean diffusivity (MD), radial diffusivity (RD), and axial diffusivity (AD) describe different aspects of diffusion and can help interpreting the results. Thus, the same analyses were performed on MD, RD, and AD (Supplement 3).

MNC. The MNC, a cohort employing more restrictive inclusion criteria, was used as a discovery sample. As the MDD patients are more homogeneous in symptom severity and treatment conditions, noise caused by such nuisance variables should be more controlled for in this cohort.

First, an ANCOVA with FA as dependent variable, diagnosis (MDD vs. HC) as independent variable and age, sex, and TIV as nuisance variables was conducted (analysis 1.1). In a subsequent analysis, total CTQ scores were included as an additional covariate to correct differences attributed to diagnosis for the influence of maltreatment. Therefore, the differences between diagnoses (MDD vs. HC) corrected for total CTQ scores, the influence of the total CTQ scores themselves and a potential $\mathrm{CTQ} \times$ diagnosis interaction were calculated (analysis 1.2). Depressed patients in the MNC and MACS differed in the Medlndex (Table 2). Therefore, additional exploratory analyses analyzing the influence of medication intake were conducted in the MDD sample alone. An ANCOVA with FA as dependent variable, Medlndex as an independent variable and age, sex, and TIV as nuisance variables was calculated (analysis 1.3).

MACS. The MACS, a cohort with more liberal recruiting strategies, was used as replication sample. Patients suffering from MDD in the MACS underwent various different treatments (inpatient, outpatient or none) and symptom severity varied more widely. While this cohort might be influenced by noise caused by these nuisance influences, it approximates the population of MDD patients to a greater extent.

In case of significant results in the MNC, significant voxels were used to mask the analyses in the MACS. Again, an ANCOVA was calculated with FA as dependent variable, diagnosis (MDD vs. HC) as independent variable and age, sex and TIV as nuisance variables masked with the significant main effect of diagnosis from analysis 1.1. This analysis was additionally adjusted for scanner differences: The body coil was exchanged mid recruitment in Marburg. To correct for the scanner differences (Marburg pre body-coil exchange, Marburg post body-coil exchange, Münster) two dummy coded variables (Marburg pre body-coil exchange: yes or no; Marburg post body coil: yes or no) with Münster as reference category were included as nuisance variables in the analysis (analysis 2.1). Similar to analysis 1.2 , total CTQ scores were then included in a second analysis masked with the significant voxels from the main effect of CTQ from the analysis 1.2 on top of age, sex, TIV, and scanner differences (Marburg pre body-coil exchange, Marburg post body-coil exchange). The main effect of diagnosis corrected for the influence of CTQ, the main effect of CTQ, and the CTQ $\times$ diagnosis interaction were calculated (analysis 2.2). Lastly, similar to analysis 1.3 , exploratory analyses for the influence of medication intake were conducted in the MDD sample alone with FA as dependent variable, Medlndex as independent variable and age, sex, TIV, and scanner differences as nuisance variables masked with the significant main effect of MedIndex from analysis 1.3 (analysis 2.3).

\section{RESULTS}

Results in the MNC

Analysis 1.1. A significant main effect of diagnosis $\left(p_{\mathrm{FWE}}=0.021\right.$, total $k=11,390$ voxels in three clusters, peak voxel of largest cluster: $x=8, y=-32, z=22$ ) was found. MDD patients had higher FA compared with $\mathrm{HC}$ in a bilateral cluster comprising the forceps minor, the superior longitudinal fasciculus and the corticospinal tract, among others (Table S1).

Analysis 1.2. This main effect of diagnosis on FA was abolished after correcting for CTQ $\left(p_{\mathrm{FWE}}=0.562\right)$. However, a significant main effect of CTQ emerged ( $p_{\mathrm{FWE}}=0.006$, total $k=32,994$ voxels in nine clusters, peak voxel of largest cluster: $x=-11, y=-6, z=$ $-6)$. FA was negatively associated with childhood maltreatment in a bilateral cluster most prominently in the anterior thalamic radiation, the corticospinal tract, the fronto-occipital fasciculi, and the longitudinal fasciculi irrespective of group (Table S2, Fig. 1). The main effect of CTQ remained significant, even after excluding potentially influential data points/outliers (Supplement 4). Further, a stepwise regression analysis of the five subtypes of childhood maltreatment showed that of the subtypes only emotional abuse and physical neglect were associated with reduced FA values (Supplement 5, Table S3). When analyzing the MDD subsample alone, the association between mean extracted FA and CTQ remained significant even after correcting for HDRS, Medlndex, comorbid diagnosis (yes vs. no) and number of hospitalizations on top of age, sex, and TIV in a subsequent analysis in SPSS (Table 3). The CTQ $\times$ diagnosis interaction was not significant $\left(p_{\mathrm{FWE}}=0.389\right)$.

Analysis 1.3. A significant increase of FA in MDD associated with higher Medlndex scores $\left(p_{\mathrm{FWE}}=0.043\right.$, total $k=2784$ voxels in four clusters, peak voxel of the largest cluster $x=-26, y=-30$, $z=27$ ) could be observed. The effect was found in the anterior thalamic radiation, the corticospinal tract, the cingulum, the forceps minor, the inferior fronto-occipital fasciculus, the longitudinal fasciculus, and the uncinate fasciculus. Patients with higher number and doses of psychopharmacological treatment had higher FA.

Results in the MACS

Analysis 2.1. The significant FA voxels described in Table S1 from the abovementioned main effect of diagnosis within the MNC (analysis 1.1) were used to mask the following 
A.
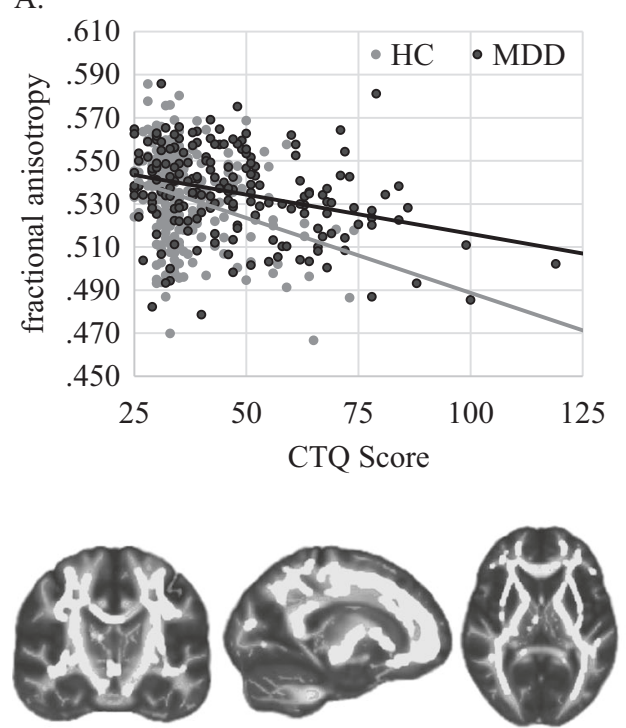

B.
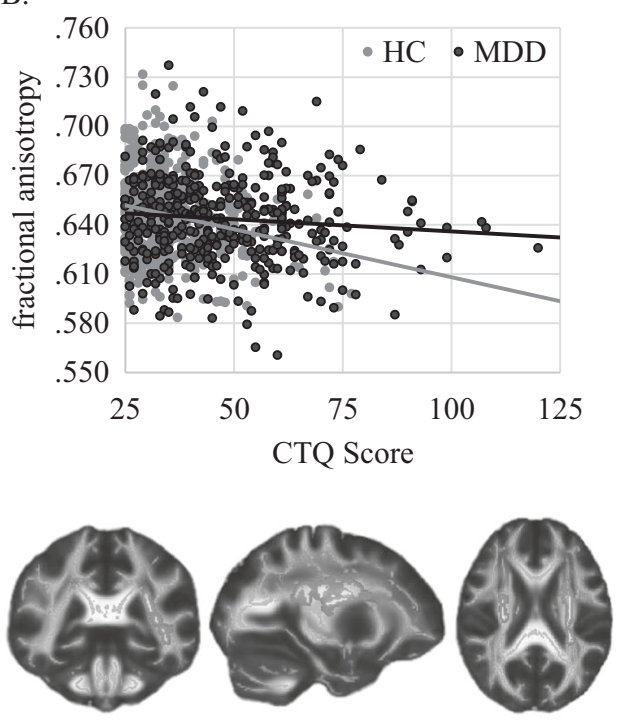

Fig. 1 Significant correlation of FA with CTQ. a Results in MNC, MNI coordinates for the section plane $x=-18, y=-17, z=6$. b Results in MACS, MNI coordinates $x=-27, y=-37, z=21$

Table 3. ANCOVA with extracted mean FA from the significant CTQ cluster as dependent variable analyzed in the MDD patients alone with age, TIV, sex, total CTQ scores, HDRS Scores, Medlndex, comorbid diagnosis (yes vs. no), and number of hospitalizations as independent variables

\begin{tabular}{|c|c|c|c|c|c|c|}
\hline & \multicolumn{3}{|l|}{ MNC } & \multicolumn{3}{|l|}{ MACS } \\
\hline & $F(1177)$ & $p$-value & Sig. & $F(1382)$ & $p$-value & Sig. \\
\hline Age & 32.14 & $<0.001$ & $* * *$ & 4.49 & 0.035 & * \\
\hline TIV & 7.08 & 0.008 & $* *$ & 16.46 & $<0.001$ & $* * *$ \\
\hline Sex & 2.10 & 0.149 & & 1.85 & 0.175 & \\
\hline CTQ & 9.81 & 0.002 & $* *$ & 4.53 & 0.034 & * \\
\hline Marburg pre body coil & - & - & - & 18.58 & $<0.001$ & $* * *$ \\
\hline Marburg post body coil & - & - & - & 21.13 & $<0.001$ & $* * *$ \\
\hline HDRS & 0.63 & 0.428 & & 0.55 & 0.459 & \\
\hline MedIndex & 1.76 & 0.186 & & 0.07 & 0.794 & \\
\hline Comorbidity & 0.42 & 0.517 & & 2.03 & 0.155 & \\
\hline Hospitalizations & 0.13 & 0.714 & & 1.98 & 0.160 & \\
\hline
\end{tabular}

CTQ Childhood Trauma Questionnaire, HDRS Hamilton, Medlndex medication load index, MACS Marburg-Münster Affective Disorders Study, MDD major depressive disorder, MNC Münster Neuroimaging Cohort, TIV Total intracranial volume

${ }^{* * *} p<0.001,{ }^{* *} p<0.01,{ }^{*} p<0.05$

corresponding analysis in the MACS sample. Neither an FA increase nor decrease in MDD compared with HC could be found (all $p_{\mathrm{FWE}}>0.212$ ). Therefore, an additional exploratory whole-brain analysis with a threshold of $p_{\text {uncorrected }}<0.05$ was conducted in the MACS sample, resulting in a marginally significant main effect of diagnosis $\left(p_{\mathrm{FWE}}=0.052 ; p_{\text {uncorrected }}=\right.$ 0.034 , total $k=939$ voxels in four clusters, peak voxel of the largest cluster $x=-22, y=-78, z=13$ ). In this cohort, MDD had lower FA compared with $\mathrm{HC}$ in the thalamic radiation, the longitudinal fasciculus, the anterior thalamic radiation, the corticospinal tract, the cingulum, the forceps major, the frontooccipital fasciculus, and the longitudinal fasciculus.
Analysis 2.2. Similarly, the significant FA voxels described in Table S2 from the abovementioned main effect of CTQ within the MNC (analysis 1.2) were used to mask the following analyses in the MACS. A significant main effect of CTQ emerged ( $p_{\mathrm{FWE}}=0.012$, total $k=1,551$ voxels in five clusters, peak voxel of largest cluster: $x=-25, y=-4, z=20$ ). Higher values of maltreatment were associated with lower FA in the corticospinal tract, the inferior fronto-occipital fasciculus and the superior and inferior longitudinal fasciculi among other regions (Table S4, Fig. 1). The main effect of CTQ remained significant even after excluding potentially influential data points/outliers (Supplement 4). A stepwise regression analysis revealed that only emotional neglect contributed to reduced FA values (Supplement 5, Table S3). The association between mean extracted FA and CTQ in the MDD group alone remained significant even after correcting for HDRS, Medlndex, comorbid diagnosis, and number of hospitalizations on top of age, sex, TIV, and scanner differences in a subsequent analysis in SPSS (Table 3). Again, there was no significant diagnosis $\times$ CTQ interaction $\left(p_{\mathrm{FWE}}=0.098\right)$ nor a main effect of diagnosis $\left(p_{\mathrm{FWE}}=0.115\right)$ present in the regions associated with CTQ in the analysis 1.2. As no significant main effect of diagnosis was found prior to the correction for CTQ, a whole-brain analysis was omitted.

Analysis 2.3. The significant voxels from the abovementioned main effect of Medlndex within the MNC's MDD patients (analysis 1.3) were used to mask the following analysis in the MACS. The significant increase of FA in MDD patients alone associated with higher values of Medlndex scores could not be replicated $\left(p_{\mathrm{FWE}}=0.678\right)$.

\section{DISCUSSION}

The aim of this study was to distinguish effects attributed to MDD from those attributed to childhood maltreatment. As expected, the differences in DTI metrics between MDD and $\mathrm{HC}$ were reduced after correcting for the influence of childhood maltreatment. Moreover, correcting for traumatic experiences sufficed to abolish all significant differences between MDD patients and HC. Furthermore, the correlation of childhood maltreatment experiences and DTI metrics did not differ between MDD patients and $\mathrm{HC}$, reflected in nonsignificant diagnosis $\times$ CTQ interactions. This 
suggests that some of the differences in DTI metrics attributed to the diagnosis of depression in previous studies might actually be due to higher levels of maltreatment among MDD patients compared with HC [24]. These results suggest that childhood maltreatment is an important confounder that should be accounted for in future studies investigating MDD. Similar results have already been described in an earlier study [52], where no differences in FA were found between MDD and HC after correcting for negative childhood experiences.

Further, the well-established finding of reduced FA following maltreatment experiences was replicated in two independent cohorts in all previously described regions. The effect was present even after correcting for common clinical characteristics (medication, comorbidities, disorder history) and remained significant even after excluding extreme values from the analysis. The present data therefore corroborate and extend previous DTI studies on FA following maltreatment experiences $[15,16,19,20]$. History of childhood maltreatment is associated with reduced $F A$ in a large bilateral network comprising the inferior fronto-occipital fasciculi, the uncinate fasciculi, the thalamic radiation, the corticospinal tract, the longitudinal fasciculi, the cingulum bundle and the corpus callosum. This effect did not seem to be driven by any specific type of maltreatment, as the association with physical neglect and emotional abuse in the MNC could not be replicated in the MACS. In the latter emotional neglect was the strongest predictor. It seems that the overall contribution of various early life events contributed to reductions in FA. Regarding a potential mechanistic explanation for the observation of reduced FA it is possible that prolonged stressful life events like childhood maltreatment result in the hypersecretion of glucocorticoids $[5,13]$ leading to altered oligodendrocyte functioning and potentially reduced myelination [53]. However, as we did not measure glucocorticoid secretion, oligodendrocyte functioning, and myelination directly, this is only speculative. Future studies should focus on the longitudinal change of FA and their biological basis to pursue this idea.

Taken together with results from structural $[26,54,55]$ and functional MRI $[25,56,57]$ these results add to evidence that childhood maltreatment is associated with changes in complex neural networks. Given the similarity between maltreated $\mathrm{HC}$ and $M D D$, it is possible that these changes reflect initially adaptive reactions to a dysfunctional environment that increase the risk for mental disorders unless compensated for [4]. This compensation could be either preexisting, e.g., genetic [58-60], or adaptive, e.g., through social support [61-63] or cognitive $[62,64]$ and behavioral differences $[65,66]$.

In the MNC, MDD patients had bilaterally higher FA compared with HC. This result is in contrast to previous analyses showing consistently that MDD patients have lower FA. Patients in the MNC had higher doses of pharmacotherapy on average compared with patients in the MACS. Medication intake was positively associated with FA in the anterior thalamic radiation, the corticospinal tract, the cingulum, the corpus callosum, the inferior fronto-occipital fasciculus, the longitudinal fasciculus, and the uncinate fasciculus in the MNC, while no association was found in the MACS. The effects of medication intake overlap partially with the effects found comparing MDD and HC prior to correction for CTQ in the MNC. Thus, the unexpected result in MDD patients in the MNC could be a consequence of pharmacotherapy. This interpretation is, however, speculative as it could not be replicated in the MACS. The influence of types and doses of antidepressant medication on FA should be explored in future studies in more detail.

Lastly, in the MACS, no significant differences between MDD and $\mathrm{HC}$ following rigorous FWE-correction were found prior to correction for CTQ. However, using uncorrected $p$-values, MDD patients showed a pattern of reduced FA in the anterior thalamic radiation, the corticospinal tract, the cingulum, the corpus callosum, the fronto-occipital fasciculus and the inferior and superior longitudinal fasciculi matching previously described results $[16-18,21-23]$. These subthreshold results in the MACS limit the interpretation that differences attributed to MDD could be traced back to higher levels of childhood maltreatment among patients. Nonetheless, a greater spectrum of MDD patients approximating the broad spectrum of depression could be recruited in the MACS. Future studies should examine to what extent FA differences can be explained by sample characteristics, e.g., remission or chronicity.

Interestingly, Choi et al. [67] found no significant differences comparing MDD patients with HC using DTI in a large sample and randomly selected subsamples as well. The authors argued that small sample sizes and tracts prone to artifacts could have resulted in false positive results in previous studies. However, they also admitted that the FA values of MDD subgroups (treatment-resistant, early trauma exposure, etc.) could differ from those of HC. Our current findings confirm the hypothesis that it is not the diagnosis of MDD itself but, rather, other aspects like childhood maltreatment experiences that could underly previously reported differences between MDD patients and $\mathrm{HC}$.

\section{Limitations}

While changes in $F A, M D, A D$, and $R D$ are linked to myelination and axonal damage, number, orientation, and crossings of axons can also influence these metrics [29]. While TBSS focuses on the tract center and uses thresholds to investigate larger tracts, this problem still arises in smaller tracts whose diameter is smaller than the voxel size [68]. Thus, future studies should employ different approaches (e.g., post mortem, animal models, High Angular Resolution Diffusion Imaging).

In this study the total CTQ score was used. While the specific contribution of any subtype of maltreatment could not be replicated, previous studies indicated that different types of maltreatment lead to different results in brain structure [4]. Hence, the different subtypes should be focused on and disentangled in future studies using DTI.

Further, a retrospective self-report questionnaire to estimate childhood maltreatment was used, which might have been influenced by a negative recall bias in MDD patients. Even though recall of childhood experiences was shown to provide reliable information in previous studies $[25,69,70]$, the retrieval of negative autobiographical memories is facilitated, faster, and less detailed in MDD patients [71-73]. While we are not aware, that childhood trauma is overreported in cohorts of MDD patients to an extent that might reduce the reliability and validity of the CTQ, the inclusion of structured interviews could have provided more reliable information [74-77].

\section{CONCLUSION}

Even though the mechanisms underlying this association are likely more complex, the similarity between $\mathrm{HC}^{\prime} \mathrm{s}$ and MDD patients' FA after controlling for negative childhood experiences suggests that some differences previously attributed to diagnosis might rather be characterized as a function of maltreatment. Reduced FA associated with more childhood maltreatment experiences was replicated using two of the largest, independent, and representative samples of patients and matched controls to this day.

\section{FUNDING AND DISCLOSURE}

Tilo Kircher received unrestricted educational grants from Servier, Janssen, Recordati, Aristo, Otsuka, neuraxpharm. This funding is not associated with the current work. The authors declare no competing interests. 


\section{ACKNOWLEDGEMENTS}

This work is part of the German multicenter consortium "Neurobiology of Affective Disorders. A translational perspective on brain structure and function", funded by the German Research Foundation (Deutsche Forschungsgemeinschaft DFG; Forschungsgruppe/Research Unit FOR2107). Principal investigators (PIs) with respective areas of responsibility in the FOR2107 consortium are: Work Package WP1, FOR2107/MACS cohort and brainimaging: Tilo Kircher (speaker FOR2107; DFG grant numbers KI 588/ 14-1, KI 588/14-2), Udo Dannlowski (co-speaker FOR2107; DA 1151/5-1, DA 1151/5-2), Axel Krug (KR 3822/5-1, KR 3822/7-2), Igor Nenadic (NE 2254/1-2), Carsten Konrad (KO 4291/3-1). WP2, animal phenotyping: Markus Wöhr (WO 1732/4-1, WO 1732/4-2), Rainer Schwarting (SCHW 559/14-1, SCHW 559/14-2). WP3, miRNA: Gerhard Schratt (SCHR 1136/3-1, SCHR 1136/3-2). WP4, immunology, mitochondriae: Judith Alferink (AL 1145/5-2), Carsten Culmsee (CU 43/9-1, CU 43/9-2), Holger Garn (GA 545/5-1, GA 545/7-2). WP5, genetics: Marcella Rietschel (RI 908/11-1, RI 908/11-2), Markus Nöthen (NO 246/10-1, NO 246/10-2), Stephanie Witt (WI 3439/3-1, WI 3439/3-2). WP6, multimethod data analytics: Andreas Jansen (JA 1890/7-1, JA 1890/7-2), Tim Hahn (HA 7070/2-2), Bertram Müller-Myhsok (MU1315/8-2), Astrid Dempfle (DE 1614/3-1, DE 1614/3-2). CP1, biobank: Petra Pfefferle (PF 784/1-1, PF 784/1-2), Harald Renz (RE 737/ 20-1, RE 737/20-2). CP2, administration. Tilo Kircher (KI 588/15-1, KI 588/17-1), Udo Dannlowski (DA 1151/6-1), Carsten Konrad (KO 4291/4-1). Data access and responsibility: All PIs take responsibility for the integrity of the respective study data and their components. All authors and coauthors had full access to all study data. Acknowledgements and members by Work Package 1: Henrike Bröhl, Bruno Dietsche, Rozbeh Elahi, Jennifer Engelen, Sabine Fischer, Jessica Heinen, Svenja Klingel, Felicitas Meier, Torsten Sauder, Annette Tittmar, Dilara Yüksel (Dept. of Psychiatry, Marburg University). Mechthild Wallnig, Rita Werner (Core-Facility Brainimaging, Marburg University). Carmen Schade-Brittinger, Maik Hahmann (Coordinating Centre for Clinical Trials, Marburg). Michael Putzke (Psychiatric Hospital, Friedberg). Rolf Speier, Lutz Lenhard (Psychiatric Hospital, Haina). Birgit Köhnlein (Psychiatric Practice, Marburg). Peter Wulf, Jürgen Kleebach, Achim Becker (Psychiatric Hospital Hephata, Schwalmstadt-Treysa). Ruth Bär (Care facility Bischoff, Neunkirchen). Matthias Müller, Michael Franz, Siegfried Scharmann, Anja Haag, Kristina Spenner, Ulrich Ohlenschläger (Psychiatric Hospital Vitos, Marburg). Matthias Müller, Michael Franz, Bernd Kundermann (Psychiatric Hospital Vitos, Gießen). Christian Bürger, Fanni Dzvonyar, Stella Fingas, Hannah Lemke, Kordula Vorspohl, Bettina Walden, Dario Zaremba (Dept. of Psychiatry, University of Münster). Harald Kugel, Jochen Bauer, Walter Heindel, Birgit Vahrenkamp (Dept. of Clinical Radiology, University of Münster). Gereon Heuft, Gudrun Schneider (Dept. of Psychosomatics and Psychotherapy, University of Münster). Thomas Reker (LWL-Hospital Münster). Gisela Bartling (IPP Münster). Ulrike Buhlmann (Dept. of Clinical Psychology, University of Münster). This work was funded by the German Research Foundation Deutsche Forschungsgemeinschaft DFG (SFBTRR58, Projects C09 and Z02 to UD) the Interdisciplinary Center for Clinical Research (IZKF) of the medical faculty of Münster (grant Dan3/012/17 to UD; SEED 11/18 to NO), IMF Münster (grant RE111604 and RE111722 to RR, RE221707 to JR, LE121703 to EJL), and the Deanery of the Medical Faculty of the University of Münster.

\section{ADDITIONAL INFORMATION}

Supplementary Information accompanies this paper at (https://doi.org/10.1038/ s41386-019-0472-y).

Publisher's note: Springer Nature remains neutral with regard to jurisdictional claims in published maps and institutional affiliations.

\section{REFERENCES}

1. Scher CD, Forde DR, McQuaid JR, Stein MB. Prevalence and demographic correlates of childhood maltreatment in an adult community sample. Child Abus Negl. 2004;28:167-180.

2. Chapman DP, Whitfield CL, Felitti VJ, Dube SR, Edwards VJ, Anda RF. Adverse childhood experiences and the risk of depressive disorders in adulthood. J Affect Disord. 2004;82:217-225.

3. Infurna MR, Reichl C, Parzer P, Schimmenti A, Bifulco A, Kaess M. Associations between depression and specific childhood experiences of abuse and neglect: $A$ meta-analysis. J Affect Disord. 2016;190:47-55.

4. Teicher MH, Samson JA, Anderson CM, Ohashi K. The effects of childhood maltreatment on brain structure, function and connectivity. Nat Rev Neurosci. 2016;17:652-666.

5. Frodl T, O'Keane V. How does the brain deal with cumulative stress? A review with focus on developmental stress, HPA axis function and hippocampal structure in humans. Neurobiol Dis. 2013;52:24-37.

6. Dube SR, Felitti VJ, Dong M, Giles WH, Anda RF. The impact of adverse childhood experiences on health problems: evidence from four birth cohorts dating back to 1900. Prev Med. 2003;37:268-277.
7. Angst J, Gamma A, Rössler W, Ajdacic V, Klein DN. Childhood adversity and chronicity of mood disorders. Eur Arch Psychiatry Clin Neurosci. 2011;261:21-27.

8. Klein DN, Arnow BA, Barkin JL, Dowling F, Kocsis JH, Leon AC, et al. Early adversity in chronic depression: clinical correlates and response to pharmacotherapy. Depress Anxiety. 2009;26:701-710.

9. Miniati M, Rucci P, Benvenuti A, Frank E, Buttenfield J, Giorgi G, et al. Clinical characteristics and treatment outcome of depression in patients with and without a history of emotional and physical abuse. J Psychiatr Res. 2010;44:302-309.

10. Nanni V, Uher R, Danese A. Childhood maltreatment predicts unfavorable course of illness and treatment outcome in depression: a meta-analysis. Am J Psychiatry. 2012;169:141-151.

11. Scott KM, McLaughlin KA, Smith DAR, Ellis PM. Childhood maltreatment and DSM-IV adult mental disorders: comparison of prospective and retrospective findings. Br J Psychiatry. 2012;200:469-475.

12. Cohen-Woods S, Fisher HL, Ahmetspahic D, Douroudis K, Stacey D, Hosang GM, et al. Interaction between childhood maltreatment on immunogenetic risk in depression: discovery and replication in clinical case-control samples. Brain Behav Immun. 2018;67:203-210.

13. Gonzalez A. The impact of childhood maltreatment on biological systems: implications for clinical interventions. Paediatr Child Health. 2013;18:415-418.

14. McCrory EJ, de Brito SA, Viding E. The impact of childhood maltreatment: a review of neurobiological and genetic factors. Front Psychiatry. 2011;2:48.

15. Choi J, Jeong B, Polcari A, Rohan ML, Teicher MH. Reduced fractional anisotropy in the visual limbic pathway of young adults witnessing domestic violence in childhood. Neuroimage. 2012;59:1071-1079.

16. Huang $\mathrm{H}$, Gundapuneedi $\mathrm{T}$, Rao $\mathrm{U}$. White matter disruptions in adolescents exposed to childhood maltreatment and vulnerability to psychopathology. Neuropsychopharmacology. 2012;37:2693-2701.

17. Lai $\mathrm{C}-\mathrm{H}, \mathrm{Wu} \mathrm{Y}-\mathrm{T}$. The white matter microintegrity alterations of neocortical and limbic association fibers in major depressive disorder and panic disorder: the comparison. Med (Baltim). 2016;95:e2982.

18. Liu X, Watanabe K, Kakeda S, Yoshimura R, Abe O, Ide S, et al. Relationship between white matter integrity and serum cortisol levels in drug-naive patients with major depressive disorder: diffusion tensor imaging study using tract-based spatial statistics. Br J Psychiatry. 2016;208:585-590.

19. McCarthy-Jones S, Oestreich LKL, Lyall AE, Kikinis Z, Newell DT, Savadjiev $P$, et al. Childhood adversity associated with white matter alteration in the corpus callosum, corona radiata, and uncinate fasciculus of psychiatrically healthy adults. Brain Imaging Behav. 2018;12:449-458.

20. Tendolkar I, Mårtensson J, Kühn S, Klumpers F, Fernández G. Physical neglect during childhood alters white matter connectivity in healthy young males. Hum Brain Mapp. 2018;39:1283-1290.

21. Choi S, Han K-M, Won E, Yoon B-J, Lee M-S, Ham B-J. Association of brain-derived neurotrophic factor DNA methylation and reduced white matter integrity in the anterior corona radiata in major depression. J Affect Disord. 2015;172:74-80.

22. Repple J, Meinert S, Grotegerd D, Kugel H, Redlich R, Dohm K, et al. A voxel-based diffusion tensor imaging study in unipolar and bipolar depression. Bipolar Disord. 2017;19:23-31.

23. Wise T, Radua J, Nortje G, Cleare AJ, Young AH, Arnone D. Voxel-based metaanalytical evidence of structural disconnectivity in major depression and bipolar disorder. Biol Psychiatry. 2016;79:293-302.

24. Widom CS, DuMont K, Czaja SJ. A prospective investigation of major depressive disorder and comorbidity in abused and neglected children grown up. Arch Gen Psychiatry. 2007;64:49-56.

25. Dannlowski U, Stuhrmann A, Beutelmann V, Zwanzger P, Lenzen T, Grotegerd D, et al. Limbic scars: long-term consequences of childhood maltreatment revealed by functional and structural magnetic resonance imaging. Biol Psychiatry. 2012;71:286-293.

26. Opel N, Redlich R, Zwanzger P, Grotegerd D, Arolt V, Heindel W, et al. Hippocampal atrophy in major depression: a function of childhood maltreatment rather than diagnosis? Neuropsychopharmacology. 2014;39:2723-2731.

27. Winston GP. The physical and biological basis of quantitative parameters derived from diffusion MRI. Quant Imaging Med Surg. 2012;2:254-265.

28. Le Bihan D. Looking into the functional architecture of the brain with diffusion MRI. Nat Rev Neurosci. 2003;4:469-480.

29. Jones DK, Knösche TR, Turner R. White matter integrity, fiber count, and other fallacies: the do's and don'ts of diffusion MRI. Neuroimage. 2013;73:239-254.

30. Repple J, Opel N, Meinert S, Redlich R, Hahn T, Winter NR, et al. Elevated bodymass index is associated with reduced white matter integrity in two large independent cohorts. Psychoneuroendocrinology. 2018;91:179-185.

31. Kircher T, Wöhr M, Nenadic I, Schwarting R, Schratt G, Alferink J, et al. Neurobiology of the major psychoses: a translational perspective on brain structure and function-the FOR2107 consortium. Eur Arch Psychiatry Clin Neurosci. 2018. https://doi.org/10.1007/s00406-018-0943-x. 
32. Vogelbacher C, Möbius TWD, Sommer J, Schuster V, Dannlowski U, Kircher T, et al. The Marburg-Münster Affective Disorders Cohort Study (MACS): a quality assurance protocol for MR neuroimaging data. Neuroimage. 2018;172:450-460.

33. Wittchen H-U, Wunderlich U, Gruschwitz S, Zaudig M, SKID I. Strukturiertes klinisches interview für DSM-IV. Achse I: psychische störungen. interviewheft und beurteilungsheft. eine deutschsprachige, erweiterte bearbeitung der amerikanischen originalversion des SKID I. Göttingen: Hogrefe; 1997.

34. Wingenfeld K, Spitzer C, Mensebach C, Grabe HJ, Hill A, Gast U, et al. The German version of the childhood trauma questionnaire (CTQ): preliminary psychometric properties. Psychother Psychosom Med Psychol. 2010;60:442-450.

35. Bernstein DP, Fink L, Handelsman L, Foote J, Lovejoy M, Wenzel K, et al. Initial reliability and validity of a new retrospective measure of child abuse and neglect. Am J Psychiatry. 1994;151:1132-1136.

36. Hamilton M. A rating scale for depression. J Neurol Neurosurg Psychiatry. 1960;23:56-62.

37. Redlich R, Almeida JJR, Grotegerd D, Opel N, Kugel H, Heindel W, et al. Brain morphometric biomarkers distinguishing unipolar and bipolar depression. a voxel-based morphometry-pattern classification approach. JAMA Psychiatry. 2014;71:1222-1230.

38. Dannlowski U, Grabe HJ, Wittfeld K, Klaus J, Konrad C, Grotegerd D, et al. Multimodal imaging of a tescalcin (TESC)-regulating polymorphism (rs7294919)-specific effects on hippocampal gray matter structure. Mol Psychiatry. 2015;20:398-404.

39. Oguz I, Farzinfar M, Matsui J, Budin F, Liu Z, Gerig G, et al. DTIPrep: quality control of diffusion-weighted images. Front Neuroinform. 2014;8:4.

40. Jenkinson M, Beckmann CF, Behrens TEJ, Woolrich MW, Smith SM. FSL. Neuroimage. 2012;62:782-790.

41. Smith SM, Jenkinson M, Woolrich MW, Beckmann CF, Behrens TEJ, Johansen-Berg $\mathrm{H}$, et al. Advances in functional and structural MR image analysis and implementation as FSL. Neuroimage. 2004;23(Suppl 1):S208-19.

42. Woolrich MW, Jbabdi S, Patenaude B, Chappell M, Makni S, Behrens TEJ, et al. Bayesian analysis of neuroimaging data in FSL. Neuroimage. 2009;45:S173-86.

43. Smith SM. Fast robust automated brain extraction. Hum Brain Mapp. 2002;17:143-155

44. Behrens TEJ, Woolrich MW, Jenkinson M, Johansen-Berg H, Nunes RG, Clare S, et al. Characterization and propagation of uncertainty in diffusion-weighted MR imaging. Magn Reson Med. 2003;50:1077-1088.

45. Smith $S M$, Jenkinson $M$, Johansen-Berg $H$, Rueckert $D$, Nichols $T E$, Mackay $C E$, et al. Tract-based spatial statistics: voxelwise analysis of multi-subject diffusion data. Neuroimage. 2006;31:1487-1505.

46. Winkler AM, Ridgway GR, Webster MA, Smith SM, Nichols TE. Permutation inference for the general linear model. Neuroimage. 2014;92:381-397.

47. Smith SM, Nichols TE. Threshold-free cluster enhancement: addressing problems of smoothing, threshold dependence and localisation in cluster inference. Neuroimage. 2009;44:83-98

48. Hua K, Zhang J, Wakana S, Jiang H, Li X, Reich DS, et al. Tract probability maps in stereotaxic spaces: analyses of white matter anatomy and tract-specific quantification. Neuroimage. 2008;39:336-347.

49. Mori S, Wakana S, van Zijl PCM, Nagae-Poetscher LM. MRI atlas of human white matter. 1st ed. Amsterdam, The Netherlands: Elsevier; 2005.

50. Wakana S, Caprihan A, Panzenboeck MM, Fallon JH, Perry M, Gollub RL, et al. Reproducibility of quantitative tractography methods applied to cerebral white matter. Neuroimage. 2007;36:630-644.

51. Zaremba D, Dohm K, Redlich R, Grotegerd D, Strojny R, Meinert S, et al. Association of brain cortical changes with relapse in patients with major depressive disorder. JAMA Psychiatry. 2018;75:484-492.

52. Ugwu ID, Amico F, Carballedo A, Fagan AJ, Frodl T. Childhood adversity, depression, age and gender effects on white matter microstructure: a DTI study. Brain Struct Funct. 2015;220:1997-2009.

53. Jauregui-Huerta F, Ruvalcaba-Delgadillo Y, Gonzalez-Castañeda R, Garcia-Estrada J, Gonzalez-Perez O, Luquin S. Responses of glial cells to stress and glucocorticoids. Curr Immunol Rev. 2010;6:195-204.

54. Frodl T, Janowitz D, Schmaal L, Tozzi L, Dobrowolny H, Stein DJ, et al. Childhood adversity impacts on brain subcortical structures relevant to depression. J Psychiatr Res. 2017;86:58-65.

55. Opel N, Zwanzger P, Redlich R, Grotegerd D, Dohm K, Arolt V, et al. Differing brain structural correlates of familial and environmental risk for major depressive disorder revealed by a combined VBM/pattern recognition approach. Psychol Med. 2016:46:277-290.

56. Dannlowski U, Kugel H, Huber F, Stuhrmann A, Redlich R, Grotegerd D, et al. Childhood maltreatment is associated with an automatic negative emotion processing bias in the amygdala. Hum Brain Mapp. 2013;34:2899-2909.

57. Redlich R, Opel N, Bürger C, Dohm K, Grotegerd D, Förster K, et al. The limbic system in youth depression: brain structural and functional alterations in adolescent in-patients with severe depression. Neuropsychopharmacology. 2018;43:546-554

58. McGowan PO, Sasaki A, D’Alessio AC, Dymov S, Labonté B, Szyf M, et al. Epigenetic regulation of the glucocorticoid receptor in human brain associates with childhood abuse. Nat Neurosci. 2009;12:342-348.

59. Tozzi L, Carballedo A, Wetterling F, McCarthy H, O'Keane V, Gill M, et al. Singlenucleotide polymorphism of the fkbp5 gene and childhood maltreatment as predictors of structural changes in brain areas involved in emotional processing in depression. Neuropsychopharmacology. 2016;41:487-497.

60. Kaufman J, Yang B-Z, Douglas-Palumberi H, Grasso D, Lipschitz D, Houshyar S, et al. Brain-derived neurotrophic factor-5-HTTLPR gene interactions and environmental modifiers of depression in children. Biol Psychiatry. 2006;59:673-680.

61. Sperry DM, Widom CS. Child abuse and neglect, social support, and psychopathology in adulthood: a prospective investigation. Child Abus Negl. 2013;37:415-425.

62. Hankin BL. Childhood maltreatment and psychopathology: prospective tests of attachment, cognitive vulnerability, and stress as mediating processes. Cogn Ther Res. 2005;29:645-671.

63. Salazar AM, Keller TE, Courtney ME. Understanding social support's role in the relationship between maltreatment and depression in youth with foster care experience. Child Maltreat. 2011;16:102-113.

64. Padilla Paredes P, Calvete E. Cognitive vulnerabilities as mediators between emotional abuse and depressive symptoms. J Abnorm Child Psychol. 2014; 42:743-753.

65. Braithwaite EC, O'Connor RM, Degli-Esposti M, Luke N, Bowes L. Modifiable predictors of depression following childhood maltreatment: a systematic review and meta-analysis. Transl Psychiatry. 2017;7:e1162.

66. Brensilver M, Negriff S, Mennen FE, Trickett PK. Longitudinal relations between depressive symptoms and externalizing behavior in adolescence: moderating effects of maltreatment experience and gender. J Clin Child Adolesc Psychol. 2011;40:607-617.

67. Choi KS, Holtzheimer PE, Franco AR, Kelley ME, Dunlop BW, Hu XP, et al. Reconciling variable findings of white matter integrity in major depressive disorder. Neuropsychopharmacology. 2014;39:1332-1339.

68. Johansen-Berg $H$, Behrens TEJ. Diffusion MRI: from quantitative measurement to in vivo neuroanatomy. 2nd ed. London: Academic Press; 2014.

69. Brewin CR, Andrews B, Gotlib IH. Psychopathology and early experience: a reappraisal of retrospective reports. Psychol Bull. 1993;113:82-98.

70. Hardt J, Rutter M. Validity of adult retrospective reports of adverse childhood experiences: review of the evidence. J Child Psychol Psychiatry. 2004;45:260-273.

71. Gotlib IH, Joormann J. Cognition and depression: current status and future directions. Annu Rev Clin Psychol. 2010;6:285-312.

72. Lloyd GG, Lishman WA. Effect of depression on the speed of recall of pleasant and unpleasant experiences. Psychol Med. 1975;5:173-180.

73. Dalgleish T, Werner-Seidler A. Disruptions in autobiographical memory processing in depression and the emergence of memory therapeutics. Trends Cogn Sci. 2014;18:596-604.

74. Lenze SN, Xiong C, Sheline YI. Childhood adversity predicts earlier onset of major depression but not reduced hippocampal volume. Psychiatry Res. 2008;162:39-49.

75. Rao U, Chen L-A, Bidesi AS, Shad MU, Thomas MA, Hammen CL. Hippocampal changes associated with early-life adversity and vulnerability to depression. Biol Psychiatry. 2010;67:357-364.

76. Baldwin JR, Reuben A, Newbury JB, Danese A. Agreement between prospective and retrospective measures of childhood maltreatment: a systematic review and meta-analysis. JAMA Psychiatry. 2019;76:584-593.

77. Goltermann J, Opel N, Dannlowski U. Considering the source of information in the evaluation of maltreatment experiences. JAMA Psychiatry. 2019. https://doi.org/10.1001/jamapsychiatry.2019.1688. 